Maturitas, 83, Jan 2016, 78-82

doi:10.1016/j.maturitas.2015.10.003

\title{
Estimating cut points: a simple method for new wearables
}

\begin{abstract}
A Hickey ${ }^{1,2}, \mathrm{~J} \mathrm{Newham}^{3}$, MM Slawinska ${ }^{4}$, D Kwasnicka ${ }^{3}, \mathrm{~S}$ McDonald ${ }^{3}$, S Del Din ${ }^{1,2}$, FF Sniehotta ${ }^{3}$, PA Davis ${ }^{4}$, A Godfrey ${ }^{1,2}$

${ }^{1}$ Institute of Neuroscience | Newcastle University Institute for Ageing, ${ }^{2}$ Clinical Ageing Research Unit, NE4 5PL, ${ }^{3}$ Institute of Health and Society, Newcastle University, Newcastle upon Tyne, NE2 $4 \mathrm{AX}, \mathrm{UK}$

${ }^{4}$ Department of Sport, Exercise and Rehabilitation, Northumbria University, Newcastle upon Tyne,
\end{abstract} NE1 8ST, UK

\footnotetext{
Corresponding author

Dr Alan Godfrey, Institute of Neuroscience, Clinical Ageing Research Unit, Newcastle University, Campus for Ageing \& Vitality, Newcastle upon Tyne, UK.

Email: alan.godfrey@ncl.ac.uk Phone: 01912081245

Fax: 01912081251
} 


\section{Abstract (247 / 250 words)}

Wearable technology is readily available for continuous assessment due to a growing number of commercial devices with increased data capture capabilities. However, many commercial devices fail to support suitable parameters (cut points) derived from the literature to help quantify PA due to differences in manufacturing. A simple metric to estimate cut points for new wearables is needed to aid data analysis.

Objective: The purpose of this pilot study was to investigate a simple methodology to determine cut points based on ratios between sedentary behaviour (SB) and PA intensities for a new wrist worn device (PRO-Diary ${ }^{\mathrm{TM}}$ ) by comparing its output to a validated and well characterised 'gold standard' $\left(\operatorname{ActiGraph}^{\mathrm{TM}}\right)$.

Study design: Twelve participants completed a semi-structured (four-phase) treadmill protocol encompassing SB and three PA intensity levels (light, moderate, vigorous). The outputs of the devices were compared accounting for relative intensity.

Results: Count ratios $(6.31,7.68,4.63,3.96)$ were calculated to successfully determine cut-points for the new wrist worn wearable technology during SB $(0-426)$ as well as light $(427-803)$, moderate $(804-2085)$ and vigorous ( $\geq 2086)$ activities, respectively.

Conclusion: Our findings should be utilised as a primary reference for investigations seeking to use new (wrist worn) wearable technology similar to that used here (i.e. Pro-Diary ${ }^{\mathrm{TM}}$ ) for the purposes of quantifying SB and PA intensities. The utility of count ratios may be useful in comparing devices or $\mathrm{SB} / \mathrm{PA}$ values estimated across different studies. However, a more robust examination is required for different devices, attachment locations and on larger/diverse cohorts.

\section{Keywords}

Cut points; sedentary behaviour; physical activity; accelerometer

\section{Highlights}

- Cut points successfully determined for a new wearable technology (PRO-Diary ${ }^{\mathrm{TM}}$ ).

- Sedentary, light, moderate \& vigorous PA cut points compare to previous literature.

- Use of count ratios may be useful to compare different devices/studies. 


\subsection{Introduction}

Wearable technology in the form of accelerometer-based body worn monitors are replacing traditional self-report measures of sedentary behaviour (SB) and physical activity (PA) [1]. Compact design and advances in computing have made devices suitable for prolonged wear time in longitudinal and/or large epidemiological studies that investigate associations between objectively measured behaviour/activity and clinical outcomes [2]. Moreover, these devices are now capable of identifying the environmental context of one's habitual sedentary/activity lifestyle [3] as well as provide ecological momentary prompts [4] for situational interventions [5]. One such wearable device is the PRO-Diary ${ }^{\mathrm{TM}}$, which incorporates a touch sensitive screen to deliver intermittent survey prompts throughout a prescribed time-period, and as such facilitates opportunities for greater participant monitoring or PA intervention within the community. However, commercial devices such as this fail to accommodate suitable parameters (cut scores/points) from the literature to differentiate SB and PA levels/intensities due to each device specification.

Objective quantification of SB and PA has been extensively investigated within the literature by utilising the (waist mounted) ActiGraph ${ }^{\mathrm{TM}}$ device which converts acceleration signals over repeated time intervals into 'epochs/counts'. Subsequently, the distinctions between SB and various PA levels (intensities) are reliant on the application of cut points [6]. The importance of appropriate cut points has been stressed due to consequent impact on data interpretation and resulting activity levels e.g. over or under-estimation of minutes of moderate to vigorous PA [3], or relationships between PA and various outcomes e.g., health, cognition [7]. The range of values used for cut points varies and relies greatly on the device specifications and a broad range of participant characteristics (age, gender, weight, etc). For example, numerous iterations (>11) of ActiGraph ${ }^{\mathrm{TM}}$ cut points have been established for young healthy subjects [8]. In order to reduce the subjectivity of activity count data analysis (epoch lengths, cut points) suggestions have been made that all prospective devices that derive PA measures from cut-offs require independent validation against other similar devices [9].

Therefore, when identifying appropriate cut-points for SB and PA intensity classification, appropriate valid continuity of new wearable technologies is paramount. This pilot study aims to investigate a simple methodology to determine SB and PA intensity cut points for a new 
accelerometer-based wearable technology (PRO-Diary ${ }^{\mathrm{TM}}$ ) by comparing it to a widely used and prevalidated activity monitoring 'gold standard' $\left(\right.$ ActiGraph $\left.^{\mathrm{TM}}\right)$ [10]. Establishing (robust) cut-points derived from a simple ratio metric will inform future SB and PA assessments for the device used here and other wearable technology new to market.

\subsection{Methods}

\subsection{Wearable technology}

The PRO-Diary $^{\mathrm{TM}}$ (camNtech Ltd. Cambridge, UK) is a small $(51 \times 34 \times 8 \mathrm{~mm})$, lightweight $(16 \mathrm{~g})$ device that was attached to the dominant wrist (predominantly right) of each participant by use of the strap attachment. The device is embedded with a tri-axial accelerometer which samples at $50 \mathrm{~Hz}$ $(0.02 \mathrm{~s})$ and was programmed to capture motion at 1 minute (60s) epochs.

(a)
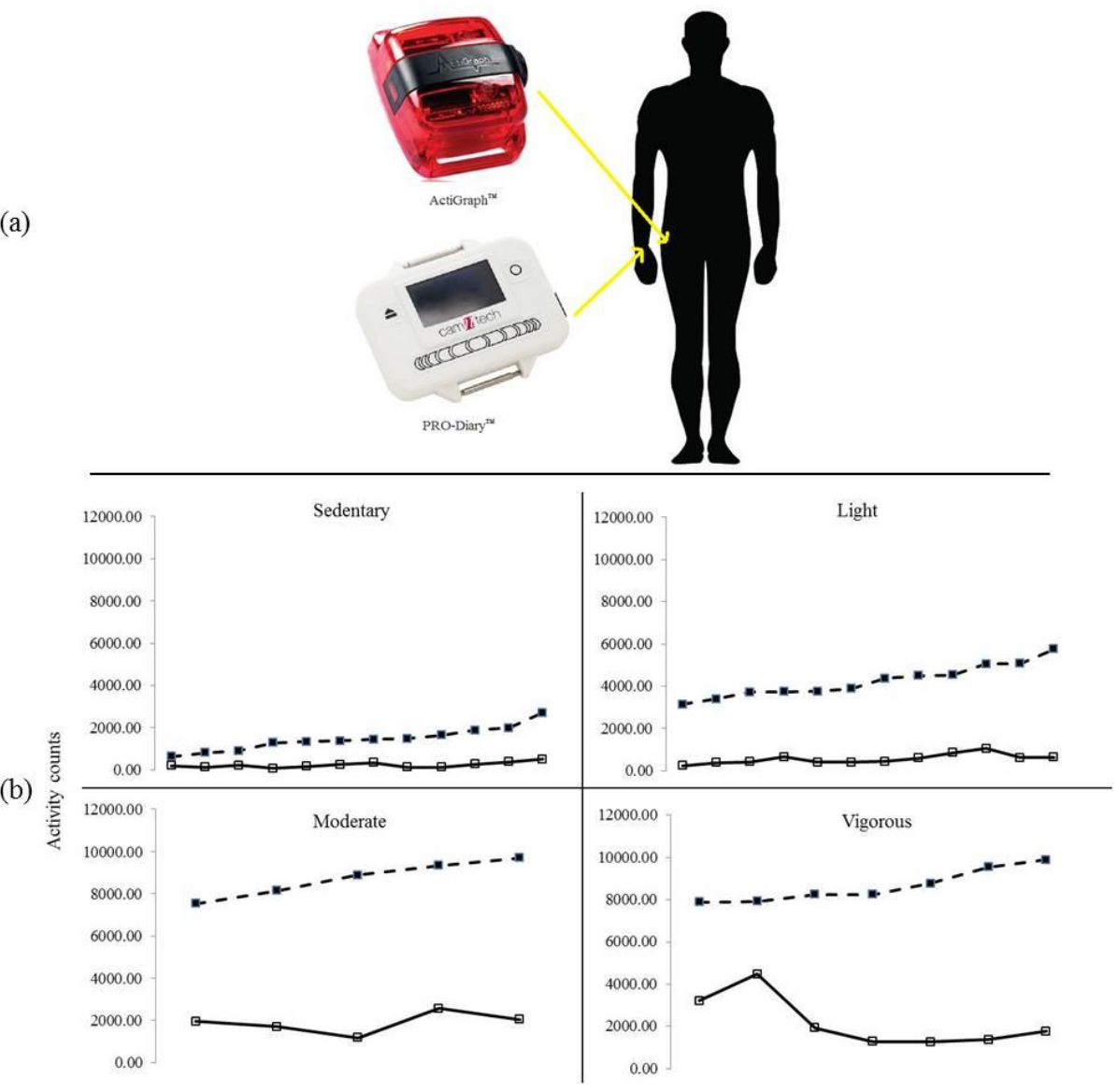

Figure 1: (a) Attachment of the ActiGraph ${ }^{\mathrm{TM}}$ (waist) and PRO-Diary ${ }^{\mathrm{TM}}$ (dominant wrist: left/right), (b) Plots demonstrating the trend between activity counts calculated by ActiGraph $^{\mathrm{TM}}$ (dashed) and PRODiary $^{\mathrm{TM}}$ (solid) for sedentary, moderate, vigorous and very vigorous physical activities 
Participants were also fitted with an ActiGraph $^{\mathrm{TM}}$ (wGT3X-BT, LLC, Penascola, US) which was taken as the reference 'gold standard' to determine PRO-Diary ${ }^{\mathrm{TM}}$ cut points. It is a small $(46 \times 33 \times$ $15 \mathrm{~mm})$, lightweight $(19 \mathrm{~g})$ device that was attached at the right waist. The ActiGraph $^{\mathrm{TM}}$ has a tri-axial accelerometer and was programmed to sample at $30 \mathrm{~Hz}$, with data processed in 1 minute epochs. Devices were synchronised and data from both devices were stored locally on the sensor's internal memory and were downloaded upon the completion of each testing session.

\subsection{Protocol}

Participants were required to complete a four stage semi-structured protocol designed to synthesise different intensities. Participants were randomly allocated to the third phase based on (i) moderate or (ii) vigorous intensity run, Table 1. Allocation was determined by participant based on participants' ventilation threshold (VT, either $20 \%$ below VT or $10 \%$ above VT) using Ekkekakis's dual mode model [11]. The treadmill-based protocol consisted of the following:

\section{Phase 1:}

Sedentary, quiet standing ( $>5$ minutes) at the beginning and end of testing

\section{Phase 2 and 4:}

Light, periods of controlled ( $\approx 5$ minutes) brisk walking at low intensity $(<5 \mathrm{~km} / \mathrm{h}, 0 \%$ treadmill gradient)

Phase 3:

(i) Moderate, run for a prolonged period (20 minutes) at high intensity $(7.0-8.0 \mathrm{~km} / \mathrm{h}, 0-$ $2 \%$ treadmill gradient).

(ii) Vigorous, run for a prolonged period (20 minutes) at highest intensity $(8.1-11.2 \mathrm{~km} / \mathrm{h}, 2$ $-4 \%$ treadmill gradient). 
Table 1: Outline of the four phase semi-structured protocol and accompanying intensity data.

\begin{tabular}{|clccc|}
\hline \multicolumn{2}{|c}{ Phase and intensity } & Time $($ Mean \pm SD) & \multicolumn{2}{c|}{ Run information (Mean \pm SD) } \\
& & $6.58 \pm 3.60$ & -- & -- \\
Speed $(\mathbf{k m} / \mathbf{h})$ & Gradient $(\%)$ \\
\hline Phase 1 & Sedentary $(\mathrm{n}=12)$ & $5.08 \pm 0.52$ & $4.80 \pm 0.00$ & 0.00 \\
Phase 2 & Light $(\mathrm{n}=12)$ & $20.00 \pm 0.00$ & $7.68 \pm 0.44$ & $0.80 \pm 1.10$ \\
Phase 3 & Moderate $(\mathrm{n}=5)$ & $18.71 \pm 3.40$ & $9.74 \pm 0.68$ & $2.86 \pm 1.07$ \\
& Vigorous $(\mathrm{n}=7)$ & $5.00 \pm 0.00$ & $4.80 \pm 0.00$ & 0.00 \\
Phase 4 & Moderate $(\mathrm{n}=12)$ & &
\end{tabular}

\subsection{Data Segmentation \& analysis}

Data were downloaded from both devices and segmented via a MATLAB ${ }^{\circledR}$ program into phases (1 -4) with the aid of time-stamps taken at testing. The program calculated the mean intensity values for each device, to determine a ratio in activity counts between ActiGraph ${ }^{\mathrm{TM}}$ and PRO-Diary ${ }^{\mathrm{TM}}$, Equation 1. The count ratio between phases were averaged to provide values that accounted for the intensity of activity that were applied to cut points from a previous methodology using the ActiGraph ${ }^{\mathrm{TM}}$ intensity values [10].

$$
\begin{array}{ll}
\text { Count ratio }=\frac{\text { ActiGraph Activity Count } \mathrm{n}}{\text { ProDiary Activity Count } \mathrm{n}} & \text { Equation } 1 \\
\text { ProDiary cut points }=\frac{\text { ActiGraph cut point values }}{\text { Count ratio }} & \text { Equation 2 }
\end{array}
$$

\subsection{Results}

Participant characteristics are presented in Table 2. There were a greater number of women participants. All participants had a body mass index (BMI) within the healthy range (18.5-24.9). Phase 3 testing for one participant was prematurely ended due to their inability to maintain intensity (vigorous: $11.2 \mathrm{~km} / \mathrm{h}, 4.0 \%$ ).

Table 2: Participant characteristics

\begin{tabular}{|lc|}
\hline Characteristic & Mean \pm SD \\
\hline Gender $(\mathrm{M} / \mathrm{F})$ & $3 / 9$ \\
Age $($ years $)$ & $28.50 \pm 4.56$ \\
Height $(\mathrm{m})$ & $1.67 \pm 0.06$ \\
Weight $(\mathrm{kg})$ & $62.38 \pm 8.12$ \\
BMI $\left(\mathrm{kg} / \mathrm{m}^{2}\right)$ & $22.37 \pm 2.19$ \\
\hline
\end{tabular}

Similar trends were observed between ActiGraph $^{\mathrm{TM}}$ and PRO-Diary ${ }^{\mathrm{TM}}$ activity counts for all participants during different intensities (Figure 1). This is reflected in the mean differences between 
counts where values increased relative to activity intensity (mean difference); sedentary (1223), light (3688), moderate (6829) and vigorous (6463).

Table 3 shows the result for the estimated PRO-Diary ${ }^{\mathrm{TM}}$ cut scores for sedentary behaviour and physical activity intensities, derived from previous ActiGraph $^{\mathrm{TM}}$ cut points and the count ratio calculated in this study.

Table 3: ActiGraph ${ }^{\mathrm{TM}}$ intensity cut-points [10], the synthesised count ratio and the derived PRODiary ${ }^{\mathrm{TM}}$ sedentary behaviour and physical activity intensity cut-points.

\begin{tabular}{|c|c|c|c|c|}
\hline \multicolumn{2}{|l|}{ ActiGraph $^{\text {TM }}$} & \multicolumn{3}{|c|}{ PRO-Diary $^{\mathrm{TM}}$} \\
\hline Relative Intensity & (CPM) & Count Ratio & Relative Intensity & $(\mathbf{C P M})$ \\
\hline No classification provided & $<2689$ & 6.31 & Sedentary & 0-426 CPM \\
\hline Moderate & $2690-6166$ & 7.68 & Light & 427-803 CPM \\
\hline Hard & $6167-9642$ & 4.63 & Moderate & 804-2085 CPM \\
\hline Very hard & $\geq 9643$ & 3.96 & Vigorous & $\geq 2086 \mathrm{CPM}$ \\
\hline
\end{tabular}

\subsection{Discussion}

This is first study to establish SB and PA intensity cut points for the new accelerometer-based wrist worn wearable technology, PRO-Diary ${ }^{\mathrm{TM}}$. This was achieved by comparing its activity counts (where possible) to a 'gold-standard' waist mounted device; ActiGraph ${ }^{\mathrm{TM}}$. This novel and simple study showed that the activity counts between devices are scalable and that cut-points can be derived by ratios in relation to pre-validated measures. This simple methodology has utility for other wearable technology where the application of established cut points is not possible due to manufacturing differences.

\subsection{Sedentary behaviour and physical activity intensities}

A key component of this exercise was to differentiate between SB and PA intensity bouts. However, we also identified cut points to quantify three PA intensities (light, moderate, vigorous). We were unable to compare our SB cut points to the recommended cut points of ActiGraph $^{\mathrm{TM}}$ due to previous limitations with that device [10]. However, a comparison of the cut points derived in this study for the PRO-Diary ${ }^{\mathrm{TM}}$ to another wrist worn device shows some similarities, where participants also performed a treadmill-based protocol at comparable speeds [12]. The referenced study calculated cut points for the Genea device worn on both wrists on a group of older adults. We observe similar cut 
points for the Genea worn on the right wrist based on similarly classified intensities (sedentary: 0 386, light: 386 - 439, moderate: 440 - 2098 and vigorous >2098), though we estimated a larger/smaller ranges for the light/moderate PA intensities, i.e. 427 - 803 / 804 - 2085, respectively. These differences could be mainly attributed to the referenced study having an older cohort (49.4 years) and differences in device functionality (sampling rate and epoch definition). However, our PA intensity ranges show greater continuity in PA intensity estimation with a more uniform spread of values (ranges) between cuts points.

\subsection{Count ratios}

An integral step in the calculation of the final cut-points has been the derivation of count ratios used to scale each targeted intensity range. The construction of a single ratio that applied to all intensities was not possible. Examination of the mean-differences suggested that as exercise intensity increased, so too did the difference between device activity counts. The non-linearity between means suggest that a single global count ratio would be insufficient to calculate all PRO-Diary ${ }^{\mathrm{TM}}$ cut-points. Therefore, it was necessary to identify specific ratios for each phase in order to more accurately differentiate between intensity ranges. The use of count ratios (such as the values derived in this pilot study) may be a useful metric in correlating SB and PA between different devices. Future research that intends to employ standardised procedures/free-living conditions should integrate the calculation steps observed in the methodology for calculation of independent count ratio values and accompanying cut-points. However, this warrants more detailed investigation in different and larger cohorts.

\subsection{Limitations}

There are limitations to applying such a robust protocol to a uniform condition such as treadmill running. Although a common tool used in performance and clinic laboratories, previous studies [13, 14] have found activity count data obtained from accelerometers on treadmills may not be identical to activity data obtained on ground. Therefore the results of this investigation may differ slightly to freeliving conditions. Future research should also seek to identify over-ground free living count ratios and 
cut-point values in a variety of age-groups to provide a general regulatory framework for all experimental settings. The limitations of differing device locations (wrist versus waist) should also be noted as a source of potential error with respect to comparing activity counts between devices. However for the purposes of this novel investigation, these limitations have been deemed acceptable.

\subsection{Conclusion}

$\mathrm{SB}$ and PA intensity cut-points for a new wrist worn wearable technology (PRO-Diary ${ }^{\mathrm{TM}}$ ) have been derived from a robust protocol while implementing current research recommendations (ActiGraph $^{\mathrm{TM}}$ cut points). Our findings should be utilised as a primary reference for investigations seeking to use any new wearable technology for the purposes of quantifying SB and PA intensities where recommended cut points from the literature cannot be applied. In order to appropriately define the use of count ratios and corresponding cut points, all wearable technology should be compared to a variety of previously validated devices, on different wearable location and in larger and diverse cohorts.

\section{Acknowledgements}

The authors would like to thank those who participated in the study.

\section{References}

1. $\quad$ Becker, B.W., The Quantified Self: Balancing Privacy and Personal Metrics. Behavioral \& Social Sciences Librarian, 2014. 33(4): p. 212-215.

2. Lee, I.M. and E.J. Shiroma, Using accelerometers to measure physical activity in large-scale epidemiological studies: issues and challenges. Br J Sports Med, 2014. 48(3): p. 197-201.

3. Gorman, E., et al., Accelerometry analysis of physical activity and sedentary behavior in older adults: a systematic review and data analysis. European Review of Aging and Physical Activity, 2014. 11(1): p. 35-49.

4. Shiffman, S., A.A. Stone, and M.R. Hufford, Ecological momentary assessment. Annu Rev Clin Psychol, 2008. 4: p. 1-32.

5. Dunton, G.F., et al., Momentary Assessment of Adults' Physical Activity and Sedentary Behavior: Feasibility and Validity. Frontiers in Psychology, 2012. 3: p. 260.

6. Montoye, A.H., et al., Evaluating the Responsiveness of Accelerometry to Detect Change in Physical Activity. Meas Phys Educ Exerc Sci, 2014. 18(4): p. 273-285.

7. Esliger, D.W. and M.S. Tremblay, Physical activity and inactivity profiling: the next generation. Can J Public Health, 2007. 98 Suppl 2: p. S195-207.

8. Kim, Y., M.W. Beets, and G.J. Welk, Everything you wanted to know about selecting the "right" Actigraph accelerometer cut-points for youth, but...: a systematic review. J Sci Med Sport, 2012. 15(4): p. 311-21. 
9. Freedson, P., D. Pober, and K.F. Janz, Calibration of accelerometer output for children. Med Sci Sports Exerc, 2005. 37(11 Suppl): p. S523-30.

10. Sasaki, J.E., D. John, and P.S. Freedson, Validation and comparison of ActiGraph activity monitors. J Sci Med Sport, 2011. 14(5): p. 411-6.

11. Ekkekakis, P., The Dual-Mode Theory of affective responses to exercise in metatheoretical context: I. Initial impetus, basic postulates, and philosophical framework. International Review of Sport and Exercise Psychology, 2009. 2(1): p. 73-94.

12. Esliger, D.W., et al., Validation of the GENEA Accelerometer. Med Sci Sports Exerc, 2011. 43(6): p. 1085-93.

13. Hendrick, P., et al., Construct validity of RT3 accelerometer: a comparison of level-ground and treadmill walking at self-selected speeds. J Rehabil Res Dev, 2010. 47(2): p. 157-68.

14. Vanhelst, J., et al., Equivalence of accelerometer data for walking and running: treadmill versus on land. J Sports Sci, 2009. 27(7): p. 669-75. 